\title{
Teleoperación Trilateral con Retardo de un Robot Móvil
}

\section{Trilateral Teleoperation with Delay of a Mobile Robot}

\section{Emanuel Slawiñski}

Instituto de Automática (INAUT). Universidad Nacional of San Juan and CONICET. Av. Libertador San Martín 1109 (oeste). J5400ARL. San Juan, Argentina slawinski@inaut.unsj.edu.ar

\section{Vicente Mut}

Instituto de Automática (INAUT). Universidad Nacional of San Juan and CONICET. Av. Libertador San Martín 1109 (oeste). J5400ARL. San Juan, Argentina

\section{Diego Daniel Santiago}

Instituto de Automática (INAUT). Universidad Nacional of San Juan and CONICET. Av. Libertador San Martín 1109 (oeste). J5400ARL. San Juan, Argentina

\section{Resumen}

Este artículo analiza la estabilidad de un sistema de teleoperación trilateral de un robot móvil. La dinámica de dos manipuladores maestros de estructura similar y un robot móvil, asimismo se tienen en cuenta retrasos variables en el tiempo y asimétricos. Un conjunto de controladores P + D se incluyen en el sistema de teleoperación con retardo. A partir del análisis realizado, se propone un procedimiento para establecer los parámetros de control con 
el fin de asegurar la estabilidad del sistema. Finalmente, el resultado teórico es verificado en la práctica por medio de pruebas sobre el sistema de teleoperación trilateral con retardo donde dos operadores humanos en colaboración y simultáneamente para conducen en un simulador 3D un robot móvil para alcanzar una tarea establecida en un entorno remoto.

Palabras Claves: teleoperación trilateral, realimentación de fuerza, controladores $P+d$, retardo de tiempo, robot móvil.

\begin{abstract}
This paper analyzes the stability of a trilateral teleoperation system of a mobile robot. The dynamics of two manipulator-like masters and mobile robot as well as time-varying and asymmetric delays are taken into account. A set of $\mathrm{P}+\mathrm{d}$ controllers is included into the delayed teleoperation system. From the analysis performed, a procedure to set the control parameters is proposed in order to assure the stability of the whole system. Finally, the theoretical result is verified in practice by means of a simple test about delayed trilateral teleoperation system, where two human operators both collaboratively and simultaneously drive a 3D simulator of a mobile robot to achieve an established task on a remote environment.
\end{abstract}

Keywords: trilateral teleoperation, force feedback, $\mathrm{P}+\mathrm{d}$ controller, time delay, mobile robot.

\title{
INTRODUCCIÓN
}

La teleoperación robots permite la ejecución de varias tareas en un entorno remoto, generalmente peligrosas y perjudiciales para los operadores humanos. Estos sistemas se utilizan para mejorar el nivel de seguridad. En los sistemas de teleoperación de robots con realimentación de fuerza, un usuario realiza tareas interactuando físicamente con el medio ambiente, donde un esclavo sigue los movimientos realizados por el maestro al mismo tiempo que el operador humano,recibe información multimodal (visual, sonido, fuerza de respuesta) [1 ]. Esta característica aumenta el campo de aplicación en varias áreas, tales como la telemedicina, la exploración, el entretenimiento, teleservicios, tele-fabricación, entre otros. Además, el uso de Internet como un canal de comunicación provoca un bajo costo para conectar los dispositivos maestro - esclavo. Sin embargo, todas las comunicaciones de Internet agregan retardos variables en el tiempo, que puede causar inestabilidad o degradación de las prestaciones [2], así como una falta de transparencia [3] del sistema de teleoperación retardado. Por lo tanto, uno de los principales problema en los sistemas de teleoperación es cómo el controlador debe estar diseñado y calibrado en presencia de retardos con el fin de asegurar un buen comportamiento en la práctica.

Respecto al estado del arte, hay muchos esquemas de control para teleoperación bilateral con retardo entre dos manipuladores [4] (y sus referencias). El concepto se utiliza comúnmente para quitar energía a fin de asegurar la estabilidad o la pasividad del sistema de circuito cerrado retardado [5]. Una de las principales propuestas incluye señales de dispersión 
o transformaciones de onda [6], en donde se mantiene la pasividad para un canal de dos puertos, transformando los retardos, en una línea de transmisión pasiva. Generalmente, las estrategias incluyen errores de coordinación entre el maestro y el esclavo. Para resolver este problema, un simple esquema de PD-like produce sistemas estables a pesar de los retrasos variables en el tiempo y asimétricos, mediante el uso de una cantidad suficiente de amortiguación inyectada en el maestro y en el esclavo [7]. Además, muchas de las estrategias diseñadas para teleoperación de manipuladores se ensayaron en teleoperación bilateral de los robots móviles, por ejemplo de control basado en la impedancia [8], el control basado en pasividad [9], y también $\mathrm{P}+\mathrm{d}$ esquemas de control [10,11]. Sin embargo, el análisis de la estabilidad es diferente por el contraste entre los modelos del maestro y el esclavo (robot móvil). Por lo tanto, un comando de posición ya no mapea en otro comando de posición, sino más bien en una referencia de velocidad a la robot móvil.

Por otro lado, varias aplicaciones, incluyendo la rehabilitación, entrenamiento quirúrgico y seguridad [12, 13], requieren de múltiples usuarios en lugar de un solo operador, para controlar el movimiento de los robots esclavos. Además, la teleoperación multilateral proporciona capacidades tales como aumento de la destreza, la mejora de la carga, la redundancia y la capacidad de manejo, entre otros. Uno de estos sistemas implican dos robot maestros y un solo robot móvil esclavo dirigido a la formación de los operadores humanos para aprender el manejo de máquinas. En este caso, la autoridad sobre el robot móvil es compartida entre los operadores que utilizan un factor dominante. Este tipo de sistema es una extensión del sistema tradicional de teleoperación bilateral, pero que incluye dos usuarios que trabajan en colaboración y en forma simultánea desde diferentes ubicaciones en un entorno remoto compartido, a través de un robot móvil para realizar una tarea determinada. En el estado del arte sobre la teleoperación de robots trilateral, existen pocos trabajos que tienen en cuenta todos los retardos de tiempo de estos sistemas. Algunos de ellos son de control basado en pasividad [14], donde se añaden los observadores y controladores de pasividad para mantener en el sistema la energía limitada; y el esquema de control difuso adaptativo[15]. Es importante señalar el resultado obtenido en [16], donde se ha propuesto una arquitectura de control trilateral para el sistema de teleoperación de doble maestro y un esclavo considerando la dinámica no lineal de robots manipuladores y retardo de tiempo variable limitado en los canales de comunicación. Este trabajo es interesante, ya que se utilizan controladores sencillos $\mathrm{P}+\mathrm{d}$, y se propone un factor de dominio explícito. Sin embargo, tal esquema de control no se puede aplicar de manera directa a la teleoperación trilateral retardada de un robot móvil, debido a que las conexiones entre los robots manipuladores y el robot móvil dificultan el análisis de la energía dentro del sistema. Es decir, la aplicación de los controladores $\mathrm{P}+\mathrm{D}$ para un teleoperación trilateral de un robot móvil requiere un nuevo análisis de estabilidad con el fin de evaluar cómo se deben establecer los parámetros de control para asegurar un comportamiento estable de todo el sistema retardado.

Este trabajo propone a esquema de control $\mathrm{P}+\mathrm{d}$, para la teleoperación trilateral con retardo de un robot móvil. La principal contribución de este trabajo implica el análisis de estabilidad, incluyendo la dinámica de dos manipuladores robóticos maestros similares y un robot móvil esclavo, así como retardos variables en el tiempo y asimétricos. Como resultado del análisis de la estabilidad se proponen los procedimientos para la calibración de los parámetros de control. Por otra parte, se muestra la teleoperación trilateral utilizando un simulador 3D con el fin de verificar el resultado obtenido a partir del análisis teórico.

El trabajo se organiza de la siguiente manera: sección II presenta algunos aspectos preliminares: tales como los modelos dinámicos empleados, y los modelos utilizados. En la 
sección III, se propone un esquema de control aplicado a la teleoperación trilateral con retardo de un robot móvil. Además, se realiza el análisis de la estabilidad en base a un Lyapunov-Krasovskii funcional (LKF). Las secciones V y VI muestran la simulación y los resultados experimentales, donde un usuario conduce un robot con ruedas. Por último, en la sección VII, se dan las conclusiones de este trabajo.

\section{PRELIMINARES}

Este trabajo analiza sistemas de teleoperación con dos operadores humanos en general uno experto y aprendiz, cada uno utiliza un dispositivo maestro (llamado maestro $1 \mathrm{y}$ el maestro 2) con el fin de manejar un robot móvil esclavo de una manera trilateral, tal como se ilustra en la figura 1.

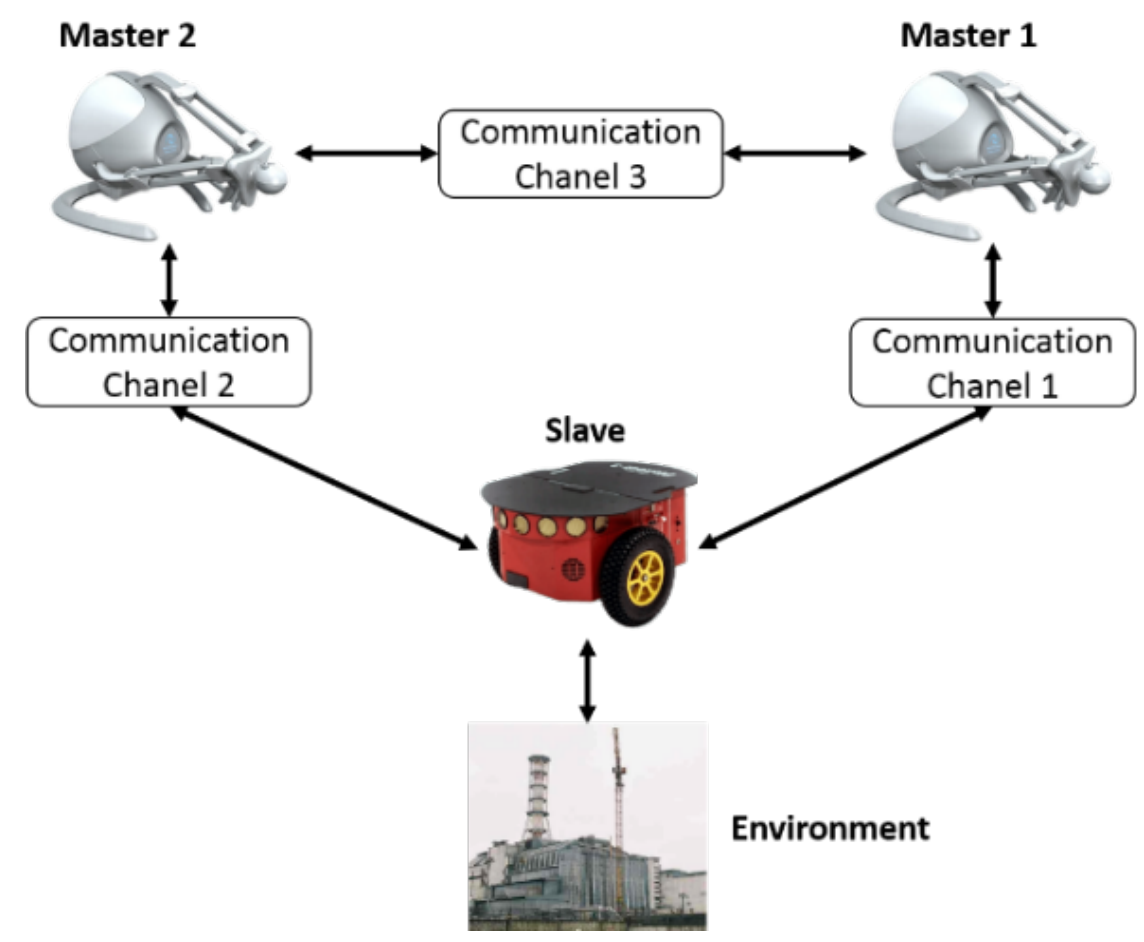

Fig. 1. Sistema de teleoperación trilateral con retardo de un robot móvil.

Cada operador humano conduce un robot manipulador para generar comandos de velocidad y también recibir retroalimentación de fuerza. El modelo dinámico no lineal estándar para representar cada robot maestro utilizado, es,

$$
\mathbf{M}_{\mathbf{m}_{i}}\left(\mathbf{q}_{\mathbf{m}_{i}}\right) \ddot{\mathbf{q}}_{\mathbf{m}_{i}}+\mathbf{C}_{\mathbf{m}_{i}}\left(\mathbf{q}_{\mathbf{m}_{j}}, \dot{\mathbf{q}}_{\mathbf{m}_{i}}\right) \dot{\mathbf{q}}_{\mathbf{m}_{j}}+\mathbf{g}_{\mathbf{m}_{i}}\left(\mathbf{q}_{\mathbf{m}_{j}}\right)=\tau_{\mathbf{m}_{j}}+\mathbf{f}_{\mathbf{h}_{j}}
$$

Donde el índice $i$ indica master 1 o master $2, \mathbf{q}_{\mathbf{m}}(t), \dot{\mathbf{q}}_{\mathbf{m}}(t) \in R^{n \times 1}$ son la posición de las 
junturas y las velocidades del maestro respectivamente; $\mathbf{M}_{\mathbf{m}}\left(\mathbf{q}_{\mathbf{m}}\right) \in R^{n \times n}$ es la matriz de inercia; $\mathbf{C}_{\mathbf{m}}\left(\mathbf{q}_{\mathrm{m}}, \dot{\mathbf{q}}_{\mathrm{m}}\right)$ es la matriz que representa los torque centrípetos y de Coriolis; $\mathbf{g}_{\mathbf{m}}\left(\mathbf{q}_{\mathbf{m}}\right)$ is el vector de torque gravitacional; $\mathbf{f}_{\mathbf{h}}$ es el torque causado por la fuerza del operador humano $\hat{\mathbf{o}}_{\mathrm{m}}$ es el torque aplicado al maestro por el controlador.

Para el caso de teleoperación de un robot móvil, el modelo dinámico de un robot móvil con tracción diferencial se considera en [17]. Tiene dos ruedas traseras accionadas independientemente y está representado por,

$$
\mathbf{D} \dot{\eta}+\mathbf{Q}(\boldsymbol{\eta}) \boldsymbol{\eta}=\tau_{\mathrm{s}}+\mathbf{f}_{\mathrm{e}}
$$

Donde $\boldsymbol{\eta}=\left[\begin{array}{ll}v & \omega\end{array}\right]^{\top}$ es el vector de las velocidades del robot con $\quad v \quad$ y $\quad \omega$ representando las velocidades lineal y angular del robot móvil, $\mathbf{f}_{\mathbf{e}}$ es la fuerza causado por el entorno sobre el robot e incluye las otras fuerzas externas no modeladas, como las fricciones estáticas y dinámicas, $\mathbf{D}=\left[\begin{array}{ll}m & 0 \\ 0 & i\end{array}\right]$ es la matriz de inercia y $\mathbf{Q}=\left[\begin{array}{cc}0 & -m a \omega \\ m a \omega & 0\end{array}\right]$ es la matriz Coriolis donde $m$ es la masa del robot, $i$ es la inercia rotacional, y $a$ es la distancia entre el centro de masa y el centro geométrico. Además, $\tau_{\mathrm{s}}=\left[\begin{array}{ll}u_{1} & u_{2}\end{array}\right]^{T}$ implican una acción de control de fuerza $u_{1}$ y una acción de control de torque $u_{2}$ las cuales se calculan a partir de la configuración de la tensión aplicada a cada motor eléctrico de corriente continua, cuya constante de tiempo se considera insignificante respecto a la constante de tiempo mecánica.

Por otra parte, el canal de comunicación se suma al retardo de tiempo hacia adelante $h_{i j}$ (desde el dispositivo i al dispositivo j) y un retardo de tiempo hacia $h_{j i}$ (desde el dispositivo $\mathrm{j}$ al dispositivo i). Los dispositivos están numerados como sigue: índice 1 representa el Master 1; 2 indica el índice maestro 2 y el índice 3 representa el robot móvil. Generalmente, estos retardos son variables en el tiempo y diferentes entre ellos (retardos asimétricos).

Por otra parte, las siguientes propiedades, suposiciones y lemas serán utilizados en el presente documento $[5,7,18]$ :

Propiedad 1: Las matrices de inercia $\mathbf{M}_{\mathbf{m}_{i}}\left(\mathbf{q}_{\mathbf{m}_{i}}\right)$ y $\mathbf{D}$ son simétricas y definidas positivas. Propiedad 2: La matriz $\dot{\mathbf{M}}_{\mathbf{m}_{i}}\left(\mathbf{q}_{\mathbf{m}_{i}}\right)-2 \mathbf{C}_{\mathbf{m}_{i}}\left(\mathbf{q}_{\mathbf{m}_{i}}, \dot{\mathbf{q}}_{\mathbf{m}_{i}}\right)$ es antisimétrica.

Propiedad 3: Existe un $k_{r}>0$ tal que $\quad \mathbf{C}_{\mathbf{m}_{i}}\left(\mathbf{q}_{\mathbf{m}_{i}}, \dot{\mathbf{q}}_{\mathbf{m}_{i}}\right) \dot{\mathbf{q}}_{\mathbf{m}_{i}} \leq k_{r}\left|\dot{\mathbf{q}}_{\mathbf{m}_{i}}\right|$ para todo tiempo . Suposición 1: Todos los retardos de tiempo $h_{i j}(t)$ y $h_{j i}(t)$ son acotados. Por lo tanto, 
existen escalares positivos $\bar{h}_{i j}$ tal que $0 \leq h_{i j}(t) \leq \bar{h}_{i j}$, para todo $t$.

Suposición 2: El operador humano tiene energía finita [5] y el entorno está representado por un modelo de amortiguación mas una perturbación de energía finita [19].

$$
\begin{aligned}
& E_{h_{i}}=\varphi_{h_{i}}-\int_{0}^{t} \mathbf{f}_{\mathbf{h}_{i}}{ }^{T} \dot{\mathbf{q}}_{\mathbf{m}_{i}} d t \geq 0 \\
& \mathbf{f}_{\mathbf{e}}=-\alpha_{e} \boldsymbol{\eta}+\mathbf{f}_{\mathbf{a}_{\mathbf{e}}}
\end{aligned}
$$

Donde $\mathbf{f}_{\mathbf{h}}$ es la fuerza del operador humano, $\varphi_{h}>0$ es el valor finite que acota la energía del operador humano, $\alpha_{e}$ es al amortiguamiento del entorno, $\mathrm{y} \mathbf{f}_{\mathbf{a}_{\mathrm{e}}}$ se supone acotada con energía finita, esto es $\left|\mathbf{f}_{\mathbf{a}_{\mathrm{e}}}\right| \leq \bar{f}_{a_{e}}$, con $\bar{f}_{a_{e}}$ una constante positiva y $\mathbf{f}_{\mathbf{a}_{\mathrm{e}}} \in L_{2}$.

Lema 1 [7]: Para funciones vectoriales reales a(.) y $\mathbf{b}($.$) y un escalar variante en el tiem-$ po $h(t)$ con $0 \leq h(t) \leq \bar{h}$, se verifica la siguiente desigualdad,

$$
-2 \mathbf{a}^{T}(t) \int_{t-h(t)}^{t} \mathbf{b}(\xi) d \xi-\int_{t-h(t)}^{t} \mathbf{b}^{T}(\xi) \mathbf{b}(\xi) d \xi \leq \bar{h}(t) \mathbf{a}^{T}(t) \mathbf{a}(t)
$$

\section{TELEOPERACIÓN TRILATERAL DE UN ROBOT MOVIL}

El sistema de teleoperación trilateral se utiliza para controlar la velocidad de un robot móvil, en el que el instructor y el aprendiz (o en general dos operadores humanos) envían comendos y perciben la fuerza realimentada. Sobre la base de esquemas de control $\mathrm{P}+\mathrm{d}$ aplicados a teleoperación bilateral de robots manipuladores [7, 18, 19] y robots móvil [10, 11], y su uso reciente en teleoperación trilateral con retardo de robots manipuladores [16], se analizará en este trabajo la estabilidad de un sistema con doble maestro y único sistema esclavo (robot móvil).

La acciones de control se proponen de la siguiente forma,

$$
\begin{aligned}
\boldsymbol{\tau}_{\mathbf{m}_{1}} & =k_{p_{1}}\left[-k_{g} \mathbf{q}_{\mathbf{m}_{1}}+\alpha \boldsymbol{\eta}\left(t-h_{31}\right)+(1-\alpha) k_{g} \mathbf{q}_{\mathbf{m}_{2}}\left(t-h_{21}\right)\right] \\
& -k_{d_{1}} \dot{\mathbf{q}}_{\mathbf{m}_{1}}+\mathbf{g}_{\mathbf{m}_{1}}\left(\mathbf{q}_{\mathbf{m}_{1}}\right) \\
\boldsymbol{\tau}_{\mathbf{m}_{2}} & =k_{p_{2}}\left[-k_{g} \mathbf{q}_{\mathbf{m}_{2}}+\alpha k_{g} \mathbf{q}_{\mathbf{m}_{1}}\left(t-h_{12}\right)+(1-\alpha) \boldsymbol{\eta}\left(t-h_{32}\right)\right] \\
& -k_{d_{2}} \dot{\mathbf{q}}_{\mathbf{m}_{2}}+\mathbf{g}_{\mathbf{m}_{2}}\left(\mathbf{q}_{\mathbf{m}_{2}}\right) \\
\boldsymbol{\tau}_{\mathbf{s}} & =k_{p_{3}}\left[\beta k_{g} \mathbf{q}_{\mathbf{m}_{1}}\left(t-h_{13}\right)+(1-\beta) k_{g} \mathbf{q}_{\mathbf{m}_{2}}\left(t-h_{23}\right)-\boldsymbol{\eta}\right] \\
& -k_{d_{3}} \mathbf{z}+\mathbf{Q}(\boldsymbol{\eta}) \boldsymbol{\eta}
\end{aligned}
$$


Donde los parámetros $k_{p_{3}}$ y $k_{d_{3}}$ son constantes positivas y representan la ganancia proporcional y la aceleración dependiente del amortiguamiento sumado por el control de velocidad ubicado en el sitio remoto, $k_{d_{1}}$ y $k_{d_{2}}$ son el amortiguamiento inyectado en los maestros, y $k_{p_{1}} \quad \mathrm{y} k_{p_{2}} \quad$ representan el un resorte relativo dependiente de la falta de sincronismo entre la posición del maestro y la referencia realimentada. Por otra parte, el parámetro $k_{g}$ mapea linealmente la posición del maestro a la velocidad de referencia, y $\mathbf{z}$ es una estimación de la aceleración del robot móvil $\dot{\eta}$.

Además, se utilizan dos factores de predominio. El factor $\alpha$, con $0<\alpha<1$, determina la autoridad del entrenador sobre el aprendiz y $1-\alpha$ implica predominio de aprendiz sobre el entrenador. Por otra parte, los factores de predominio $\beta$ y $1-\beta$ indican la supremacía del entrenador y el aprendiz sobre el robot móvil respectivamente. La relación entre ellos se define como sigue:

$$
\beta=\frac{\alpha^{2}}{\alpha^{2}+(1-\alpha)^{2}}
$$

\section{ESTABILIDAD DEL SISTEMA DE LAZO CERRADO CON RETARDO}

El análisis de estabilidad del sistema de control se basa en la teoría de Lyapunov-Krasovskii aplicado a teleoperación trilateral de un robot móvil en presencia de retardos de tiempo. Un

funcional $\mathrm{V}(\mathbf{x})=\sum_{i=1}^{8} \mathrm{~V}_{i}>0$ se propone para analizar su evolución a lo largo de las trayecto${ }^{\text {ria: }} \mathbf{x}:=\left[\begin{array}{lllllll}\dot{\mathbf{q}}_{\mathrm{m}_{1}} & \dot{\mathbf{q}}_{\mathrm{m}_{2}} & \boldsymbol{\eta} & \mathbf{z} & \boldsymbol{\eta}-k_{g} \mathbf{q}_{\mathrm{m}_{1}} & \boldsymbol{\eta}-k_{\mathrm{g}} \mathbf{q}_{\mathrm{m}_{2}} & k_{g} \mathbf{q}_{\mathrm{ml}}-k_{\mathrm{g}} \mathbf{q}_{\mathrm{m}_{2}}\end{array}\right]^{I}$

A continuación cada $\mathrm{V}_{i}$ se representa matemáticamente como sigue:

$$
\begin{aligned}
& \mathrm{V}_{1}=1 / 2 \alpha k_{p_{3}} k_{p_{1}}{ }^{-1} \dot{\mathbf{q}}_{\mathbf{m}_{1}}{ }^{\mathrm{T}} \mathbf{M}_{\mathbf{m}_{1}}\left(\mathbf{q}_{\mathbf{m}_{1}}\right) \dot{\mathbf{q}}_{\mathbf{m}_{1}}+\alpha k_{p_{3}} k_{p_{1}}{ }^{-1} E_{k_{1}} \\
& \mathrm{~V}_{2}=\frac{1}{2}(1-\alpha) \frac{k_{p_{3}}}{k_{p_{2}}} \dot{\mathbf{q}}_{\mathbf{m}_{2}}{ }^{\mathrm{T}} \mathbf{M}_{\mathbf{m}_{2}}\left(\mathbf{q}_{\mathrm{m}_{2}}\right) \dot{\mathbf{q}}_{\mathrm{m}_{2}}+(1-\alpha) \frac{k_{p_{3}}}{k_{p_{2}}} E_{b_{3}} \\
& \mathrm{~V}_{3}=1 / 2\left[\alpha^{2}+(1-\alpha)^{2}\right] \alpha_{e} k_{g}{ }^{-1} \boldsymbol{\eta}^{\mathrm{T}} \boldsymbol{\eta}
\end{aligned}
$$




$$
\begin{aligned}
& \mathrm{V}_{4}=\left[\alpha^{2}+(1-\alpha)^{2}\right] k_{g}^{-1} \int_{0}^{t} \dot{\boldsymbol{\eta}}^{\mathrm{T}} \mathbf{D} \dot{\boldsymbol{\eta}} \\
& \mathrm{V}_{5}=1 / 2 \alpha^{2} k_{g}^{-1} k_{p_{3}}\left(\boldsymbol{\eta}-k_{g} \mathbf{q}_{\mathbf{m}_{1}}\right)^{\mathbf{T}}\left(\boldsymbol{\eta}-k_{g} \mathbf{q}_{\mathbf{m}_{1}}\right) \\
& \mathrm{V}_{6}=1 / 2(1-\alpha)^{2} k_{p_{3}} k_{g}^{-1}\left(\boldsymbol{\eta}-k_{g} \mathbf{q}_{\mathrm{m}_{2}}\right)^{\mathrm{T}}\left(\boldsymbol{\eta}-k_{g} \mathbf{q}_{\mathrm{m}_{2}}\right) \\
& \mathrm{V}_{7}=1 / 2 \alpha(1-\alpha) k_{p_{3}} k_{g}{ }^{-1}\left(k_{g} \mathbf{q}_{\mathrm{m} 1}-k_{g} \mathbf{q}_{\mathrm{m}_{2}}\right)^{\mathbf{T}}\left(k_{g} \mathbf{q}_{\mathrm{m} 1}-k_{g} \mathbf{q}_{\mathrm{m}_{2}}\right) \\
& \mathrm{V}_{8}=\alpha(1-\alpha) k_{\rho_{3}} k_{g} \int_{-h_{12} t}^{0} \int_{t=0}^{t} \dot{\mathbf{q}}_{\mathbf{m}_{1}}{ }^{T} \dot{\mathbf{q}}_{\mathbf{m}_{1}}(\xi) \mathbf{d} \xi+\alpha^{2} k_{p_{3}} \int_{-h_{31} 1+0}^{0} \int_{t+0}^{t} \mathbf{z}^{T} \mathbf{z}(\xi) \mathbf{d} \xi \\
& +\alpha(1-\alpha) k_{p_{3}} k_{g} \int_{-h_{21}}^{0} \int_{i+0}^{t} \dot{\mathbf{q}}_{\mathbf{m}_{2}}{ }^{T} \dot{\mathbf{q}}_{\mathbf{m}_{2}}(\xi) \mathbf{d} \xi+(1-\alpha)^{2} k_{p_{3}} \int_{-h_{22}}^{0} \int_{t=0}^{t} \mathbf{z}^{T} \mathbf{z}(\xi) \mathbf{d} \xi \\
& +\alpha^{2} k_{p_{3}} \int_{-h_{13}}^{0} \int_{t+\theta}^{t} \dot{\mathbf{q}}_{\mathbf{m}_{1}}{ }^{T} \dot{\mathbf{q}}_{\mathbf{m}_{1}}(\xi) \mathbf{d} \xi+(1-\alpha)^{2} k_{p_{3}} \int_{-h_{2}=}^{0} \int_{t+\theta}^{t} \dot{\mathbf{q}}_{\mathbf{m}_{2}}{ }^{T} \dot{\mathbf{q}}_{\mathbf{m}_{2}}(\xi) \mathbf{d} \xi
\end{aligned}
$$

Para obtener $\dot{\mathrm{V}}$, se debe computar la derivada de las ecuaciones (8) a (15).

El sub funcional $\dot{\mathrm{V}}_{1}$ se obtiene de las ecuaciones (8),(1),(6) y aplicando las Propiedades 1 y 2.

$$
\begin{aligned}
\dot{\mathrm{V}}_{1}= & -\alpha \frac{k_{p_{3}}}{k_{p_{1}}} k_{d_{1}} \dot{\mathbf{q}}_{\mathbf{m}_{1}}{ }^{\mathrm{T}} \dot{\mathbf{q}}_{\mathbf{m}_{1}}+\alpha^{2} k_{p_{3}} \dot{\mathbf{q}}_{\mathbf{m}_{1}}{ }^{\mathrm{T}} \mathbf{\eta} \\
& +\alpha(1-\alpha) k_{p_{3}} k_{g} \dot{\mathbf{q}}_{\mathbf{m}_{1}}{ }^{\mathrm{T}} \mathbf{q}_{\mathbf{m}_{2}}-\alpha^{2} k_{p_{3}} \dot{\mathbf{q}}_{\mathbf{m}_{1}}{ }^{\mathrm{T}} \int_{t-h_{31}}^{t} \mathbf{z}(\xi) d \xi \\
& -\alpha k_{p_{3}} k_{g} \dot{\mathbf{q}}_{\mathrm{m}_{1}}{ }^{\mathrm{T}} \mathbf{q}_{\mathbf{m}_{1}}-\alpha(1-\alpha) k_{p_{3}} k_{g} \dot{\mathbf{q}}_{\mathbf{m}_{1}}{ }^{\mathrm{T}} \int_{t-k_{21}}^{t} \dot{\mathbf{q}}_{\mathbf{m}_{2}}(\xi) d \xi
\end{aligned}
$$

$\dot{\mathrm{V}}_{2}$ se computa utilizando la derivada temporal de (9), las ecuaciones (1), (6) and las Propiedades 1 y 2. 


$$
\begin{aligned}
\dot{\mathrm{V}}_{2}= & -(1-\alpha) \frac{k_{p_{3}}}{k_{p_{2}}} k_{d_{2}} \dot{\mathbf{q}}_{\mathbf{m}_{2}}{ }^{\mathbf{T}} \dot{\mathbf{q}}_{\mathbf{m}_{2}}+(1-\alpha)^{2} k_{p_{3}} \dot{\mathbf{q}}_{\mathbf{m}_{2}}{ }^{\mathrm{T}} \boldsymbol{\eta} \\
& +\alpha(1-\alpha) k_{p_{3}} k_{g} \dot{\mathbf{q}}_{\mathbf{m}_{2}}{ }^{\mathbf{T}} \mathbf{q}_{\mathbf{m}_{1}}-(1-\alpha) k_{p_{3}} k_{g} \dot{\mathbf{q}}_{\mathbf{m}_{2}}{ }^{\mathbf{T}} \mathbf{q}_{\mathbf{m}_{2}} \\
& -(1-\alpha) \alpha k_{p_{3}} k_{g} \dot{\mathbf{q}}_{\mathbf{m}_{2}}{ }_{t-h_{12}}^{\mathbf{T}} \dot{\mathbf{q}}_{\mathbf{m}_{1}}(\xi) d \xi \\
& -(1-\alpha)^{2} k_{p_{3}} \dot{\mathbf{q}}_{\mathbf{m}_{2}}{ }_{t-h_{32}}^{\mathrm{T}} \int_{t}^{t} \mathbf{z}(\xi) d \xi
\end{aligned}
$$

Luego, $\dot{\mathrm{V}}_{3}$ se obtiene desde (10)

$$
\dot{\mathrm{V}}_{3}=\left[\alpha^{2}+(1-\alpha)^{2}\right] k_{g}{ }^{-1} \alpha_{e} \boldsymbol{\eta}^{\mathrm{T}} \dot{\boldsymbol{\eta}}
$$

Además $\quad \dot{\mathrm{V}}_{4}$ se computa desde (11), la dinámica del robot móvil (2), la acción de control (6), y el modelo del entorno (4) y $\beta$ (7):

$$
\begin{aligned}
\dot{\mathrm{V}}_{4} & =-\left[\alpha^{2}+(1-\alpha)^{2}\right]\left(k_{g}{ }^{-1} \alpha_{e}-k_{p_{3}}\right) \dot{\boldsymbol{\eta}}^{\mathrm{T}} \boldsymbol{\eta} \\
& +\left[\alpha^{2}+(1-\alpha)^{2}\right] k_{g}^{-1}\left(\mathbf{z}^{\mathrm{T}} \mathbf{p}_{\mathbf{e}}-k_{d_{3}} \mathbf{z}^{\mathrm{T}} \mathbf{z}\right) \\
& +\alpha^{2} k_{p_{3}} \dot{\boldsymbol{\eta}}^{\mathrm{T}} \mathbf{q}_{\mathbf{m}_{1}}+(1-\alpha)^{2} k_{p_{3}} \dot{\boldsymbol{\eta}}^{\mathrm{T}} \mathbf{q}_{\mathbf{m}_{2}} \\
& -\alpha^{2} k_{p_{3}} \mathbf{z}^{\mathrm{T}} \int_{t-h_{13}}^{t} \dot{\mathbf{q}}_{\mathbf{m}_{1}}(\xi) d \xi-(1-\alpha)^{2} k_{p_{3}} \mathbf{z}^{\mathbf{T}} \int_{t-h_{23}}^{t} \dot{\mathbf{q}}_{\mathbf{m}_{2}}(\xi) d \xi
\end{aligned}
$$

Luego, desde (12), (13) y (14) se obtienen las siguientes ecuaciones:

$$
\begin{aligned}
& \dot{\mathrm{V}}_{5}=\alpha^{2} k_{p_{3}}\left[k_{g}{ }^{-1} \mathbf{c}^{T} \dot{\boldsymbol{c}}-\mathbf{c}^{T} \dot{\mathbf{q}}_{\mathbf{m}_{1}}-\mathbf{q}_{\mathbf{m}_{1}}{ }^{T} \dot{\boldsymbol{c}}+k_{g} \mathbf{q}_{\mathbf{m}_{1}}{ }^{T} \dot{\mathbf{q}}_{\mathbf{m}_{1}}\right] \\
& \dot{\mathrm{V}}_{6}=(1-\alpha)^{2} k_{p_{3}}\left[k_{g}{ }^{-1} \boldsymbol{\eta}^{T} \dot{\boldsymbol{\eta}}-\boldsymbol{\eta}^{T} \dot{\mathbf{q}}_{\mathbf{m}_{2}}-\mathbf{q}_{\mathbf{m}_{2}}{ }^{T} \dot{\boldsymbol{\eta}}+k_{g} \mathbf{q}_{\mathbf{m}_{2}}{ }^{\mathrm{T}} \dot{\mathbf{q}}_{\mathbf{m}_{2}}\right] \\
& \dot{\mathrm{V}}_{7}=\alpha(1-\alpha) k_{p_{3}} k_{g}\left[\mathbf{q}_{\mathbf{m} 1}{ }^{T} \dot{\mathbf{q}}_{\mathbf{m} 1}-\mathbf{q}_{\mathbf{m} 1}{ }^{T} \dot{\mathbf{q}}_{\mathbf{m} 2}-\mathbf{q}_{\mathbf{m} 2}{ }^{T} \dot{\mathbf{q}}_{\mathbf{m} 1}+\mathbf{q}_{\mathbf{m} 2}{ }^{T} \dot{\mathbf{q}}_{\mathbf{m} 2}\right]
\end{aligned}
$$

Por otra parte, $\dot{\mathrm{V}}_{8}$ se deduce desde (15) (ver [18] y [10] para mas detalles) como sigue: 


$$
\begin{aligned}
\dot{\mathrm{V}}_{8} \leq & \alpha^{2} k_{p_{3}} \bar{h}_{31} \mathbf{z}^{T} \mathbf{z}-\alpha^{2} k_{p_{3}} \int_{t-h_{31}}^{t} \mathbf{z}^{T} \mathbf{z}(\xi) d \xi \\
& +(1-\alpha) \alpha k_{p_{3}} k_{g} \bar{h}_{12} \dot{\mathbf{q}}_{\mathbf{m}_{\mathbf{1}}}{ }^{T} \dot{\mathbf{q}}_{\mathbf{m}_{\mathbf{1}}}+\alpha(1-\alpha) k_{p_{3}} k_{g} \bar{h}_{21} \dot{\mathbf{q}}_{\mathbf{m}_{2}}{ }^{T} \dot{\mathbf{q}}_{\mathbf{m}_{2}} \\
& -(1-\alpha) \alpha k_{p_{3}} k_{g} \int_{t-h_{12}}^{t} \dot{\mathbf{q}}_{\mathbf{m}_{1}}{ }^{T} \dot{\mathbf{q}}_{\mathbf{m}_{\mathbf{1}}}(\xi) d \xi \\
& -\alpha(1-\alpha) k_{p_{3}} k_{g} \int_{t-h_{21}}^{t} \dot{\mathbf{q}}_{\mathbf{m}_{2}}{ }^{T} \dot{\mathbf{q}}_{\mathbf{m}_{2}}(\xi) d \xi \\
& +(1-\alpha)^{2} k_{p_{3}} \bar{h}_{32} \mathbf{z}^{T} \mathbf{z}_{-}(1-\alpha)^{2} k_{p_{3}} \int_{t-h_{32}}^{t} \mathbf{z}^{T} \mathbf{z}(\xi) d \xi \\
& +\alpha^{2} k_{p_{3}} \bar{h}_{13} \dot{\mathbf{q}}_{\mathbf{m}_{1}}{ }^{T} \dot{\mathbf{q}}_{\mathbf{m}_{1}}-\alpha^{2} k_{p_{3}} \int_{t-h_{13}}^{t} \dot{\mathbf{q}}_{\mathbf{m}_{1}}{ }^{T} \dot{\mathbf{q}}_{\mathbf{m}_{1}}(\xi) d \xi \\
& +(1-\alpha)^{2} k_{p_{3}} \bar{h}_{23} \dot{\mathbf{q}}_{\mathbf{m}_{2}}{ }^{T} \dot{\mathbf{q}}_{\mathbf{m}_{2}}-(1-\alpha)^{2} k_{p_{3}} \int_{t-h_{23}}^{t} \dot{\mathbf{q}}_{\mathbf{m}_{2}}{ }^{T} \dot{\mathbf{q}}_{\mathbf{m}_{2}}(\xi)
\end{aligned}
$$

A continuación se unen las funciones $\dot{\mathrm{V}}_{i}$, varios términos se cancelan entre si, Si se aplica el Lema 2 (5) a los términos integrales, se obtiene el siguiente resultado:

$$
\dot{\mathrm{V}} \leq \dot{\mathbf{q}}_{\mathbf{m}_{1}}{ }^{\mathbf{T}} \lambda_{1} \mathbf{I} \dot{\mathbf{q}}_{\mathbf{m}_{1}}+\dot{\mathbf{q}}_{\mathbf{m}_{2}}{ }^{\mathbf{T}} \lambda_{2} \mathbf{I} \dot{\mathbf{q}}_{\mathbf{m}_{2}}+\mathbf{z}^{\mathbf{T}} \lambda_{3} \mathbf{I} \mathbf{z}+\mathbf{p}_{\mathbf{e}}{ }^{\mathbf{T}} \mathbf{p}_{\mathbf{e}}
$$

Con

$$
\begin{aligned}
\lambda_{1}= & {\left[4^{-1} \alpha(1-\alpha) k_{p_{3}} k_{g}\left(\bar{h}_{12}+\bar{h}_{21}\right)\right.} \\
& \left.-\alpha k_{p_{3}} k_{p_{1}}{ }^{-1} k_{d_{1}}+\alpha^{2} k_{p_{3}}\left(\bar{h}_{13}+4^{-1} \bar{h}_{31}\right)\right] \\
\lambda_{2}= & {\left[-(1-\alpha) k_{p_{3}} k_{p_{2}}{ }^{-1} k_{d_{2}}+4^{-1} \alpha(1-\alpha) k_{p_{3}} k_{g}\left(\bar{h}_{21}+\bar{h}_{12}\right)\right.} \\
& \left.+4^{-1}(1-\alpha)^{2} k_{p_{3}}\left(\bar{h}_{23}+\bar{h}_{32}\right)\right] \\
\lambda_{3}= & {\left[-\left[\alpha^{2}+(1-\alpha)^{2}\right] k_{d_{3}} k_{g}^{-1}+\alpha^{2} k_{p_{3}} 4^{-1}\left(\bar{h}_{13}+\bar{h}_{31}\right)\right.} \\
& \left.+\left(2 k_{g}\right)^{-2}+4^{-1}(1-\alpha)^{2} k_{p_{3}}\left(\bar{h}_{23}+\bar{h}_{32}\right)\right]
\end{aligned}
$$


se integra entre $0 \mathrm{yt}$, se obtiene:

$$
\mathrm{V}(t)-\mathrm{V}(0) \leq \lambda_{1}\left\|\dot{\mathbf{q}}_{\mathbf{m}_{1}}\right\|_{2}^{2}+\lambda_{2}\left\|\dot{\mathbf{q}}_{\mathbf{m}_{2}}\right\|_{2}^{2}+\lambda_{3}\|\mathbf{z}\|_{2}^{2}+\left\|\mathbf{f}_{\mathbf{e}_{\mathbf{a}}}\right\|_{2}^{2}
$$

Resultado 1. De (24), si los coefecientes de amortiguamiento $k_{d_{1}}, k_{d_{2}}, k_{d_{3}}$ son suficientemente altos tales que $\lambda_{1}, \lambda_{2}, \lambda_{3}<0$, entonces

$$
\mathrm{V}(t) \leq V(0)+\left\|\mathbf{f}_{\mathrm{e}_{\mathrm{a}}}\right\|_{2}^{2}
$$

Es decir, dado que $\mathbf{f}_{\mathbf{e}_{\mathrm{a}}} \in L_{2}$ (Suposición 2), y el functional $\mathrm{V}(\mathbf{x})$ es radialmente no acotado y desde (25) también es acotado para todo, entonces $\mathbf{x} \in L_{\infty}$. Además, teniendo en cuenta este resultado en (24), obtenemos:

$$
\lambda_{1}\left\|\dot{\mathbf{q}}_{\mathbf{m}_{1}}\right\|_{2}^{2}+\lambda_{2}\left\|\dot{\mathbf{q}}_{\mathbf{m}_{2}}\right\|_{2}^{2}+\lambda_{3}\|\mathbf{z}\|_{2}^{2} \leq\left\|\mathbf{f}_{\mathbf{a}_{\mathbf{e}}}\right\|_{2}^{2}+V(0)
$$

Esto significa que las variables $\dot{\mathbf{q}}_{\mathrm{m}_{1}}, \dot{\mathbf{q}}_{\mathrm{m}_{2}}, \mathbf{z} \in L_{2}$.

Resultado 2. From the result achieved in (23), it is possible to infer that if $k_{d_{1}}, k_{d_{2}}, k_{d_{3}}$ are greater, then $\dot{\mathbf{q}}_{\mathbf{m}_{1}}, \dot{\mathbf{q}}_{\mathrm{m}_{2}}, \mathbf{z}$ (both master velocities and slave acceleration) will tend to a smaller convergence ball.

\section{RESULTADOS EXPERIMENTALES}

En esta sección se prueba el esquema de control propuesto. Dos operadores humanos teleoperan un robot móvil, y reciben realimentación visual y de fuerza. En los dispositivos maestros se emplean manipuladores de bajo, modelo Falcon Novint http://www.novint.com que cuenta con 3 grados de libertad y realimentación de fuerza. Dos DOF de cada dispositivo maestro se utiliza para generar comandos de velocidad para mover el robot: una para la velocidad lineal y el otro para establecer la referencia de velocidad angular. Para poner en práctica el sistema de teleoperación, se utilizan las siguientes herramientas: MATLAB / Simulink de www.mathworks.com funcionando con el módulo de tiempo real, y la biblioteca SAS del

https://drive.google.com/folderview?id=0B7pAxnD2G3sOQXgwdUUyRTV1Qzg\&usp= compatible con el entrono de V-REP (http://www.coppeliarobotics.com). La metodología utilizada permite hacer pruebas de un sistema de teleoperación trilateral con retardo usando un robot simulado pero teniendo en cuenta los dos operadores humanos dentro del sistema, que se llama comúnmente simulaciones human-in-the-loop, como se muestra en la Fig. 2. La comunicación entre los dispositivos maestros, Simulink y V-REP se realiza a través de memoria compartida. 


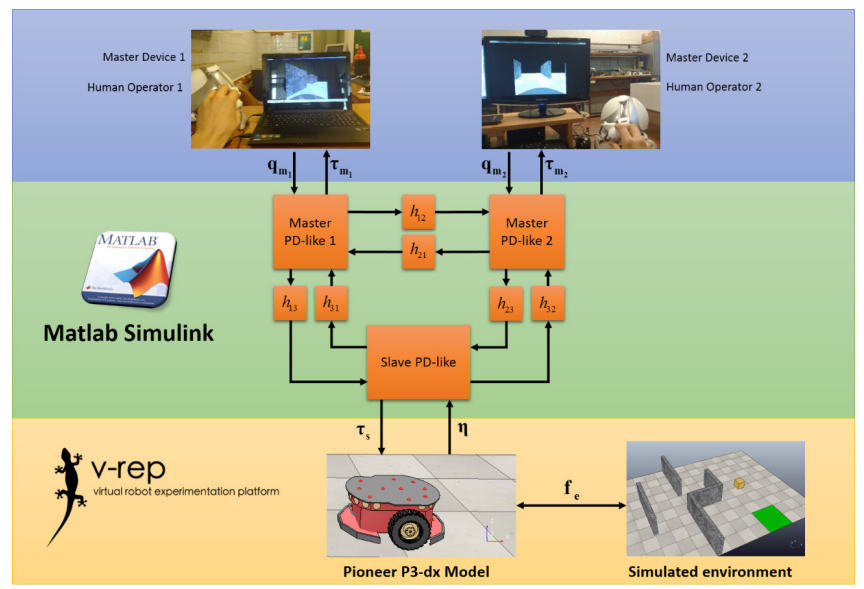

Fig 2. Sistema de teleoperación bilateral con retardo implementado para las pruebas

Para las pruebas, todos los retardos de tiempo $h_{i j}$ se configuran aleatoriamente con valores desde 0.35 seg. a 0.65 seg., mientras los parámetros de control son calibrados siguiendo el criterio de estabilidad:

$$
\begin{aligned}
& \alpha=0.6 k_{g}=10 ; k_{p_{1}}=15 ; k_{p_{2}}=15 ; k_{p_{3}}=\operatorname{diag}\left(\left[\begin{array}{ll}
200 & 35
\end{array}\right]\right) \\
& k_{d_{1}}=100 ; k_{d_{2}}=100 ; k_{d_{3}}=1
\end{aligned}
$$

El objetivo del experimento es navegar evitando las paredes para alcanzar el cubo amarillo. A continuación, el cubo amarillo debe ser empujado desde su posición inicial al sitio pintado de verde, como se muestra en la Figura 3. Ambos operadores sienten no sólo el movimiento ejercido por el otro operador sino también la dinámica del robot móvil y el peso de la caja.

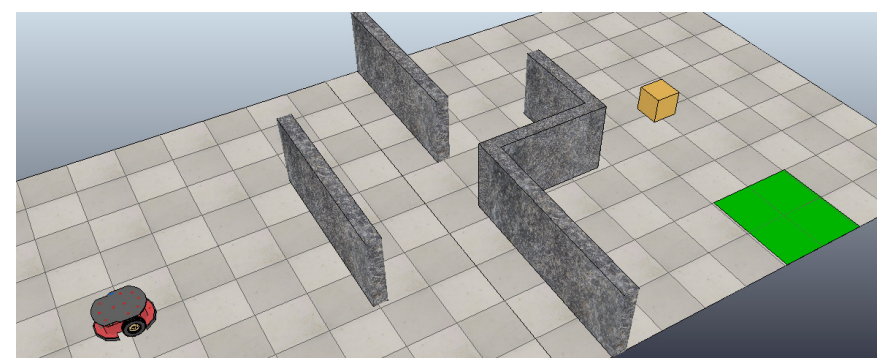

Fig. 3 Estructura de la prueba.

La Fig. 4 muestra la evolución en el sitio remoto de cada componente de las referencias (de velocidad lineal y angular) $k_{g} \mathbf{q}_{\mathrm{m}_{1}}\left(t-h_{13}\right)$ y $k_{g} \mathbf{q}_{\mathbf{m}_{1}}\left(t-h_{13}\right)$ y la velocidad del robot móvil $\eta$ (donde es la velocidad lineal y $\omega$ es la velocidad angular). Puede verse que la velocidad 
del robot móvil está más cerca del maestro 1 debido a $\alpha=0.6$ y a partir de (7) obtenemos $\beta \approx 0.7$. Esta valor establece el nivel de prioridad sobre el robot móvil. La Fig. 5 muestra una secuencia de imágenes capturadas de la prueba, donde es posible observar que la tarea se lleva a cabo adecuadamente a pesar de los retardos agregados por el canal de comunicación trilateral.
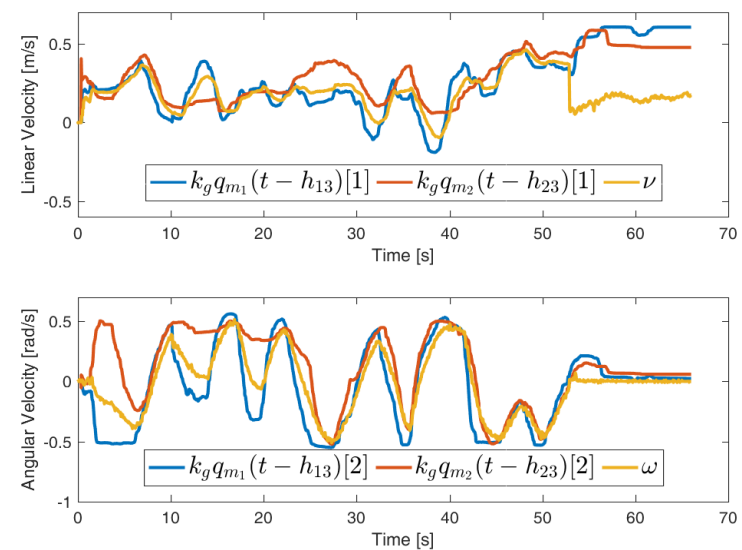

Fig. 4 Velocidades lineal y angular del robot móvil y las referencias duales de los maestros 1 y 2.

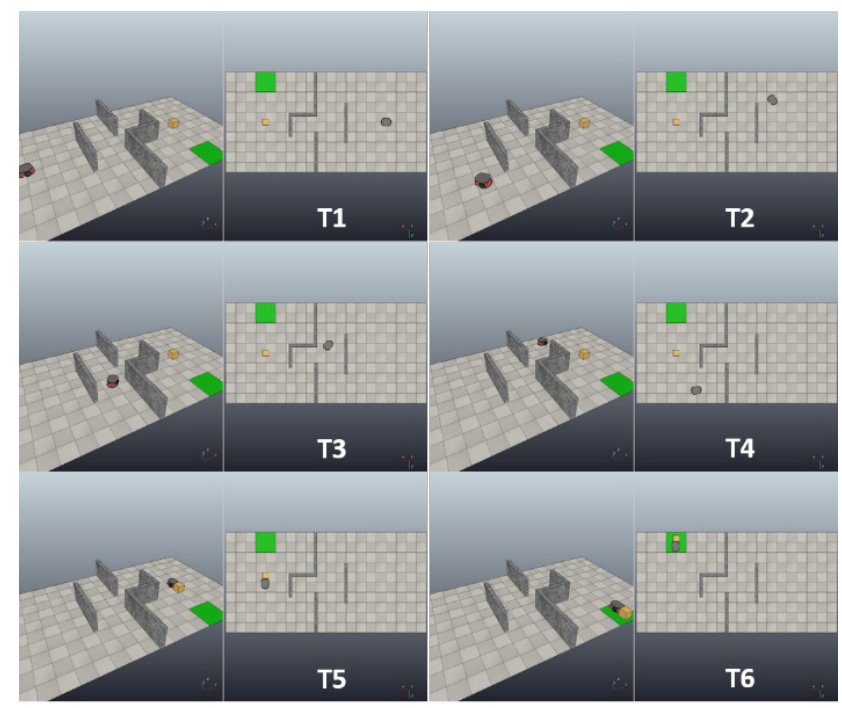

Fig. 5 Secuencias de imágenes denominadas T1, T2, T3, T4, T5 y T6 capturadas en el sitio remoto.

Finalmente en las Figuras 6 y 7, se muestran las principals señales del Sistema acerca de su estabilidad. Tal como se demostró en el análisis teórico (Resultados 1 y 2), los errores de sincronismo entre: maestro 1 y maestro $2 \mathbf{e}_{3}=k_{g} \mathbf{q}_{\mathbf{m}_{1}}(t)-k_{g} \mathbf{q}_{\mathbf{m}_{2}}(t)$, maestro 1 y robot móvil 
$\mathbf{e}_{1}=k_{g} \mathbf{q}_{\mathbf{m}_{1}}(t)-\boldsymbol{\eta}(t)$, and maestro 2 y robot móvil $\mathbf{e}_{2}=k_{g} \mathbf{q}_{\mathrm{m}_{2}}(t)-\boldsymbol{\eta}(t)$, así como la aceleración del robot móvil $\mathbf{z}$, velocidad del maestro $1 \dot{\mathbf{q}}_{\mathrm{m}_{1}}$ y velocidad del maestro $2 \dot{\mathbf{q}}_{\mathbf{m}_{2}}$, permanecen acotados a lo largo de las pruebas. Es importante señalar que alrededor de los 53 segundos (T5 en la Fig. 5) el robot móvil, impulsado por los dos operadores humanos, comienza a empujar la caja

Es posible apreciar esta acción en las señales $\mathbf{e}_{1}$ y $\mathbf{e}_{2}$ (Fig. 7) así como en la aceleración del robot $\mathbf{z}$ (Fig. 6). Es decir, dos operadores humanos teleoperando un robot móvil en forma simultánea y colaborativa para cumplir una tarea requerida en un entorno remoto.
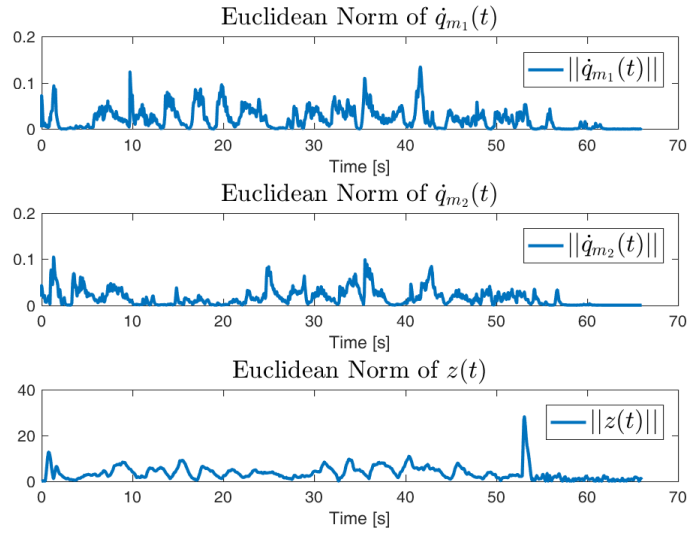

Fig. 6. Velocidades de ambos maestros y aceleración del robot móvil.

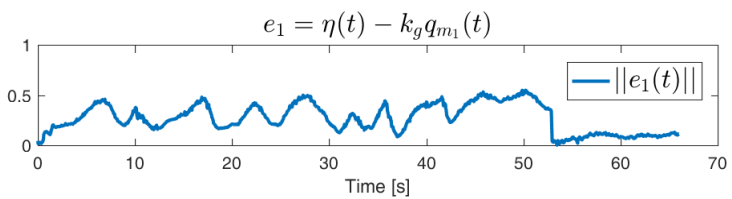

$e_{2}=\eta(t)-k_{g} q_{m_{2}}(t)$

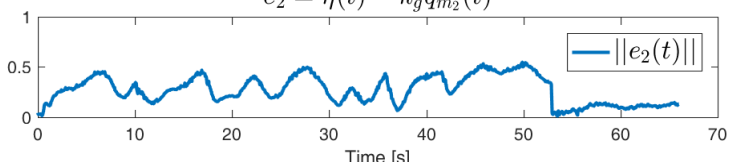

$e_{3}=k_{g} q_{m_{1}}(t)-k_{g} q_{m_{2}}(t)$

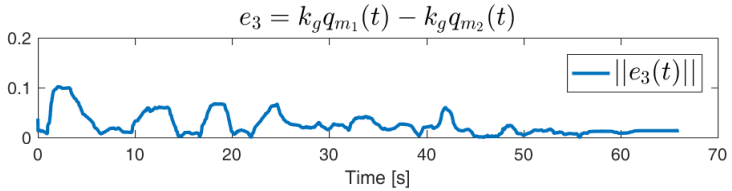

Fig. 7. Errores de sincronismo. 


\section{CONCLUSIONES}

En este documento, la estabilidad de un sistema de teleoperación trilateral con retardo de un robot mediante un sistema maestro con dos manipuladores se ha llevado a cabo. El desajuste en el mapeo entre el robot móvil y los manipuladores maestros, hace que el análisis presentado sea diferente con respecto a la aplicada a los robots manipuladores. Como resultado del análisis de la estabilidad expuesto, se proponen los procedimientos para la calibración de los parámetros, con el fin de acotar las principales señales del sistema con retardo. Además, se muestra una prueba simple sobre teleoperación trilateral utilizando un simulador 3D, cuyo resultado está de acuerdo con el resultado obtenido a partir del análisis teórico. El campo actual aplicación principal es la formación a distancia de los operadores para conducir diferentes tipos de máquinas móviles o sistemas robóticos móviles tales como manipuladores móviles colaborativos. 


\section{REFERENCIAS}

[1] T. B. Sheridan, Telerobotics, automation, and human supervisory control. Cambridge, MA, USA: MIT press, 1992.

[2] T. A. Varkonyi, I. J. Rudas, P. Pausits, and T. Haidegger, "Survey on the control of time delay teleoperation systems," in IEEE 18th International Conference on Intelligent Engineering Systems INES 2014, Tihany, Hungary, 2014, pp. 89-94.

[3] D. A. Lawrence, "Stability and transparency in bilateral teleoperation," IEEE transactions on robotics and automation, vol. 9, pp. 624-637, 1993.

[4] R. Muradore and P. Fiorini, "A review of bilateral teleoperation algorithms," Acta Polytech. Hung., vol. 13, pp. 191-208, 2016.

[5] E. Nuño, L. Basañez, and R. Ortega, "Passivity-based control for bilateral teleoperation: A tutorial," Automatica, vol. 47, pp. 485-495, 2011.

[6] G. Niemeyer and J.-J. Slotine, "Stable adaptive teleoperation," IEEE Journal of Oceanic Engineering, vol. 16, pp. 152-162, 1991.

[7] E. Nuno, R. Ortega, N. Barabanov, and L. Basanez, "A globally stable PD controller for bilateral teleoperators," IEEE Transactions on Robotics, vol. 24, pp. 753-758, 2008.

[8] I. Farkhatdinov, J.-H. Ryu, and J. An, "A preliminary experimental study on haptic teleoperation of mobile robot with variable force feedback gain," in Haptics Symposium, 2010 IEEE, 2010, pp. 251-256.

[9] H. Van Quang, I. Farkhatdinov, and J.-H. Ryu, "Passivity of delayed bilateral teleoperation of mobile robots with ambiguous causalities: Time domain passivity approach," in Intelligent Robots and Systems (IROS), 2012 IEEE/RSJ International Conference on, 2012, pp. 2635-2640.

[10] E. Slawiñski, V. Mut, and D. Santiago, "PD-like controller for delayed bilateral teleoperation of wheeled robots," International Journal of Control, pp. 1-24, 2016.

[11] D. D. Santiago, E. Slawiñski, and V. A. Mut, "Stable Delayed Bilateral Teleoperation of Mobile Manipulators," Asian Journal of Control, 2017.

[12] P. R. Culmer, A. E. Jackson, S. Makower, R. Richardson, J. A. Cozens, M. C. Levesley, et al., "A control strategy for upper limb robotic rehabilitation with a dual robot system," IEEE/ASME Transactions on Mechatronics, vol. 15, pp. 575-585, 2010.

[13] K. Shamaei, L. H. Kim, and A. M. Okamura, "Design and evaluation of a trilateral shared-control architecture for teleoperated training robots," in Engineering in Medicine and Biology Society (EMBC), 2015 37th Annual International Conference of the IEEE, 2015, pp. 4887-4893.

[14] M. Panzirsch, J. Artigas, A. Tobergte, P. Kotyczka, C. Preusche, A. Albu-Schaeffer, et al., "A peer-to-peer trilateral passivity control for delayed collaborative teleoperation," Haptics: Perception, Devices, Mobility, and Communication, pp. 395-406, 2012.

[15] Z. Li, L. Ding, H. Gao, G. Duan, and C.-Y. Su, "Trilateral teleoperation of adaptive fuzzy force/motion control for nonlinear teleoperators with communication random 
delays," IEEE transactions on Fuzzy Systems, vol. 21, pp. 610-624, 2013.

[16] A. Ghorbanian, S. Rezaei, A. Khoogar, M. Zareinejad, and K. Baghestan, "A novel control framework for nonlinear time-delayed dual-master/single-slave teleoperation," ISA transactions, vol. 52, pp. 268-277, 2013.

[17] D. Lee, O. Martinez-Palafox, and M. W. Spong, "Bilateral teleoperation of a wheeled mobile robot over delayed communication network," in Proceedings 2006 IEEE International Conference on Robotics and Automation, ICRA 2006. , 2006, pp. 3298-3303.

[18] C.-C. Hua and X. P. Liu, "Delay-dependent stability criteria of teleoperation systems with asymmetric time-varying delays," IEEE Transactions on Robotics, vol. 26, pp. 925-932, 2010.

[19] S. Islam, P. X. Liu, A. El Saddik, J. Dias, and L. Seneviratne, "Bilateral shared autonomous systems with passive and nonpassive input forces under time varying delay," ISA transactions, vol. 54, pp. 218-228, 2015. 